\title{
(6) Rare Myopathies
}

\author{
Eijiro Satoyoshi, M D
}

The Fourth Department of Medicine, Ohashi Hospital, Toho University School of Medicine

The most of classical neuromuscular disorders was established in the latter half of the 19 th century, although over a dozen of new diseases have been found for the last 3 decades based on the progress of biological, biochemical and histochemical knowledge.

In muscular dystrophies, out of the classical Duchenne dystrophy, fascioscapulohumeral dystrphy and limb-girdle type, distal myopathy, ocular myopathy and oculopharyngeal dystophy were described. Oculo-pharyngo-distal myopathy was proposed by our investigation. This type was late-onset myopathy, transmitted by autosomal dominant inheritance and characterized by slowly progressive external opthalmoplegia, distal involvement of muscles. Kerns-Shy syndrome, simulating progressive ophthalmoplegia, is characterized by ophthalmoplegia, retinal pigmentation, progressive proximal muscle atrophy, elevated CSF protein, optic atrophy, complete heart block, cerebellar symptoms, neuropathy, pyramidal signs, mental retardation and others, and may fall into a category of generalized, probably metabolic disorder, of which pathogenesis is not established.

A striking progress has been taken place concerning the congenital myopathy which is characterized by non-progressive floppy musculature since birth. Congenital muscular dystrophy proposed by Fukuyama is characterized by generalized muscular atrophy and weakness, mental retardation and epileptic seizure, and pathological study established micropolygyri as well as muscular dystrophy. It should be called a new entity of "cerebro-muscular disorders.
Kongenitale atonisch-sclerotische Muskel Dystrophie, proposed by Ullrich in 1930, is also a rare myopathy. Central core disease found by Shy and Magee in 1956 was the first step of discovery of many new congenital myopathies which are based on the congenital abnormalities of muscle fiber structure. This myopathy is characterized by central core in muscle fibers in which enzyme activities were absent. Myotubular myopathy, found by Spiro and Shy, is also characterized by central nuclei surrounded by perinuclear myopathic changes. Clinically ocular and facial involvement as well as generalized muscular atrophy is a feature. Nemaline myopathy, found Shy, is characterized by long face, high arched palate, pigeon breast, arachnodactry similar to Marfan's syndrome, and contained numerous nemaline rods in muscle fibers. Congenital fiber type dysproportion is based on the congenital abnormal distribution of diffuse type I fiber atrophy and clinically close to nemaline myopathy. These congenital myopathies may be called "congenital neuro-myopathy" because numerous abnormalities are accompanied in the skeletal and nervous system.

Numerous metabolic myopathies have been found in the last two decades some of which pathogenesis is not clarified untill present though some of congenital myopathies might be included in this group in near future.

Since the discovery of muscle phosphorylase deficient myopathy by McArdle in 1951, attention has been paid by many reseachers and some of unkown myopathies were found to belong to the cabohydrate 
metabolism disorder. Acid maltase deficiency due to complete abcense of amylo 1,4 glucosidase, was found in adult life and clinically resembling a picture of limbgirdle muscular dystrophy or polymyositis with cardiomegalia. Mc-Ardle's disease is characterized by painful contracture under anerobic exercise and lack of blood lactate formation due to a block of anerobic glycolysis. Similar symptoms may appear by the block of various glycolytic pathways. Tarui and others first described phosphofructokinase deficiency and Satoyoshi also reported the cases showing block at the level of phosphohexoisomerase level.

Lipid storage myopathy was found recently by Engel and others. Clinically progressive proximal muscle weakness and atrophy including facial and pharyngeal muscles, responded well to predonisone treatment. Lipid droplets consisting of mostly triglyceride in type I fibers was found to be due to carnitine deficiency. Luft disease charaterized by hypermetabolism, hyperthermia, polyuria, and perspiration is based on the block of junction between oxidative phosphorylation and respiration in mitochondria which are enlarged and increased in number and figures. Malignant hyperthermia myopathy is chatacterized by hyperthermia and generalized muscle contraction and break down, propagated by halothene and succinylcholine during anesthesia. Periodic paralysis is a rather common muscle disease in Japan. Most of these were hypokalemic type and recently hyperkalemic periodic paralysis or combination of hyper and hypo- kalemic periodic paralysis in the same patient, were reported infrequently. Both hyper-and hypo-kalemic paralysis may be a difference of clinical expression of the same disorder which is based on the abnormality of cell membrane permiability.

Polymyositis is a kind of collagen or auto-immune disorders. However many unkown myopathies may be included because of clinical similarities. In cases of chronic polymyositis several atypical muscle involvement were reported such as "quadriceps myopathy”, distal myositis, or fascioscapulohumeral type. Chronic segmental myopathy reported in our department was clinically close to polymyositis, though histologically segmental destruction of muscle fibers with numerous cytoplasmic bodies beneath the intact cell membrane was a specific feature.

Eaton-Lambert syndrome, so-called myasthenic syndrome, is a disease of neuromuscular junction and increment of strength by exercise and distal weakness and poor response to anticholine-esterase drugs. There may be some other myasthenic symptoms resembling to myasthenia gravis and different from pictures.

In summary, some of rare myopathies are described clinically and histopathologically. Many of the newly found myopathies were reported anywhere in Japan. However there are many unkown neuromuscular disorders which were undiagnosed or undifferentiated from present knowledges. This short presentation may be a guidance of the recent progress of new and rare muscular disorders. 